\title{
Deterioro cognitivo en ancianos diabéticos hospitalizados en medicina interna en un hospital de segundo nivel de atención en México, Distrito Federal
}

\author{
María de los Ángeles Adareli Coronel Chacón,* Beatriz Carmona Mejía, ** Gandhy Ponce Gómez***
}

\begin{abstract}
RESUMEN
Introducción: La OMS estima que a nivel mundial existen 250 millones de personas con diabetes, de las cuales, en México, por cada 100,000 corresponden 372, con una mayor prevalencia en ancianos, lo que incrementa por dos veces el riesgo de deterioro cognitivo (DC) después de seis años de evolución. El profesional de enfermería tiene como una de sus funciones asistir a la persona y a su familiar para afrontar estas alteraciones de la salud y así prevenir un daño mayor. Métodos: Es un estudio cuantitativo, descriptivo, observacional y prospectivo, con muestra por conveniencia: 100 diabéticos hospitalizados, mayores de 65 años, a quienes se aplicaron las escalas: Mini-Mental de Folstein (MMSE) y el cuestionario de polifarmacia. La tabulación fue a través de SPSS 19.0. Resultados: El 39\% de la población tiene entre 66 y 70 años; predominaron las mujeres en un 65\%; el 90\% tiene más de seis años de evolución con diabetes; $51 \%$ presentó polifarmacia. El $81 \%$ obtuvo un nivel de deterioro cognitivo, mientras que el 19\% muestra un nivel de cognición normal. El 43\% obtuvo un nivel leve de deterioro cognitivo y el $22 \%$ un nivel moderado; el 19\% tuvo un nivel normal de cognición y el 16\% muestra un nivel grave de deterioro cognitivo. Discusión: El 79\% tiene una evolución de más de seis años. El 81\% de la población padece deterioro cognitivo, lo cual está relacionado con diabetes y alto riesgo de demencia. La diabetes mellitus tipo 2 agrava condiciones que se asocian con la dependencia funcional, como lo es el deterioro cognitivo. Esta investigación pretende ser una base para desarrollar intervenciones de enfermería enfocadas a valorar el estado cognitivo de los ancianos diabéticos hospitalizados y para elevar la calidad de vida con la participación del cuidador primario.
\end{abstract}

Palabras clave: Deterioro cognitivo, diabetes mellitus tipo 2, ancianos.

\section{Cognitive impairment in diabetic elderly hospitalized in internal medicine in a hospital for a second level of care in Mexico, Distrito Federal}

\begin{abstract}
Introduction: The WHO estimates that globally there are 250 million people with diabetics, which in México are per 100,000 372 with a higher prevalence in elderly in Mexico, diabetes mellitus type 2 (DMT2) the leading cause of mortality increase by two times the risk of cognitive impairment after six years of the evolution. The nurse is one of his duties and individual and assist individuals and their family in adapting to the changes in health and prevention of further damage. Methods: The study is quantitative, descriptive, observational and prospective, with convenience sample: 100 people with diabetes hospitalized, over 65 years old, which scales were applied: Folstein Mini-Mental (MMSE) and the questionnaire of polypharmacy. The tabulation was via SPSS 19.0. Results: The 39\% of the population is between 66 and 70 years, women
\end{abstract}

* Lic. en Enfermería, Facultad de Estudios Superiores Zaragoza, UNAM.

** Prof. Carrera Asoc. C. Def., Facultad de Estudios Superiores Zaragoza, UNAM.

*** Prof. Carrera Asoc. C. Def., Unidad de Investigación, Escuela Nacional de Enfermería y Obstetricia, UNAM.

Correspondencia: María de los Ángeles Adareli Coronel Chacón. Calle Felipe Obregón Núm. 19, Col. Paraje San Juan, Delegación Iztapalapa, 09830, México, D.F. Teléfono: 5518203287

Este artículo puede ser consultado en versión completa en http://www.medigraphic.com/enfermerianeurologica 
predominated in $65 \%, 90 \%$ have more than six years of disease onset, $51 \%$ had polypharmacy. The $81 \%$ obtained a level of cognitive impairment while $19 \%$ have a level of normal cognition. The $43 \%$ had a mild level of cognitive impairment, $22 \%$ had moderate, $19 \%$ had a normal cognition and $16 \%$ have a severe level of cognitive impairment. Discussion: The $79 \%$ has evolved over six years, and therefore cognitive changes. With regard to cognitive impairment, $81 \%$ of the population has cognitive impairment is related to diabetes and high risk of dementia. Diabetes mellitus type 2 leads to agravate conditions that are associated with functional dependence as the cognitive impairment. This research seeks to develop a basis for nursing interventions aimed to assess the cognitive status of elderly hospitalized diabetics, to improve the quality of life with the participation of primary caregiver.

Key words: Cognitive impairment, diabetes mellitus type II, elderly.

\section{INTRODUCCIÓN}

\section{Antecedentes e importancia}

$\mathrm{E}$ n México, de acuerdo con las estadísticas del Instituto Nacional de Estadística, Geografía e Informática (INEGI), mientras la población general crece $1.7 \%$ anual, la población de adultos mayores se incrementa $2.5 \%$, elevándose de esta manera las enfermedades cronicodegenerativas. En México, la diabetes mellitus tipo 2 (DMT2) es la primera causa de morbimortalidad debido a sus complicaciones. Esta enfermedad ocasiona alteraciones estructurales bioquímicas de los vasos sanguíneos, por lo que incrementa por dos veces el riesgo de que el paciente tenga deterioro cognitivo, principalmente después de seis años de evolución de la enfermedad. ${ }^{1}$

El deterioro cognitivo (DC) es una disminución de las capacidades mentales referentes a la adquisición, organización, retención y uso del conocimiento. La influencia de la diabetes mellitus tipo 2 en el DC ha sido estudiada desde 1922; existe evidencia desde hace tres décadas de esta correlación. En la práctica hospitalaria diaria este factor no es evaluado en forma sistemática por los profesionales de la salud, lo cual agrava el problema de los pacientes, disminuyendo su calidad de vida. $^{2}$

Enfermedades cronicodegenerativas como la diabetes mellitus producen daño a órganos y sistemas del cuerpo humano durante su transcurso, pero esta enfermedad, en específico, origina complicaciones que afectan ojos, riñones, corazón y extremidades inferiores en quien la padece; este tipo de complicaciones son las que mayormente han sido investigadas; sin embargo, existen otras que han recibido poca atención, que pueden aparecer por la hiperglucemia crónica o por la comorbilidad que tienen con la hipertensión arterial, y que además repercuten de manera significativa en el sistema nervioso central (SNC) por las alteraciones estructurales y bioquímicas en los vasos sanguíneos, provocando, en consecuencia, el deterioro o la declinación de las funciones cognitivas. ${ }^{3}$
Los trastornos cognitivos vinculados con el envejecimiento se han convertido en un problema importante de salud. En el ámbito nacional, los trabajos realizados con respecto al deterioro cognitivo han mostrado cifras de prevalencia en la comunidad de 4.2 a $19.6 \% .^{4,5}$ El DC constituye una condición de alto riesgo para la aparición de la demencia. ${ }^{6}$

Como se ha visto, tanto los padecimientos crónicos como el deterioro cognitivo han sido asociados con el envejecimiento; no obstante, otras investigaciones realizadas por diversos autores señalan una asociación existente entre la diabetes mellitus y el deterioro cognitivo, como la realizada por Hassing, donde se concluyó que los pacientes con diabetes mostraron una importante declinación cognitiva, no así los pacientes con hipertensión sin diabetes. Sin embargo, el deterioro mayor se detectó en los portadores de ambas patologías. En este estudio se hace referencia a que los cambios cognitivos se perciben más fácilmente en pacientes ancianos con diabetes mellitus después de seis años, en comparación con los no portadores. ${ }^{7}$

Canazaro y otros autores realizaron un estudio a 254 ancianos mayores de 60 años, con el objetivo de evaluar los déficits cognitivos; los resultados mostraron una diferencia significativa en el desempeño de los ancianos con diabetes tipo 2 en comparación con el grupo control, ya que los diabéticos mostraron mayor deterioro cognitivo. ${ }^{8}$

\section{MÉTODOS}

El diseño del estudio es cuantitativo, descriptivo, observacional y prospectivo, con el objeto de identificar el nivel de deterioro cognitivo en pacientes ancianos diabéticos hospitalizados en medicina interna, en una unidad de segundo nivel de atención de México, Distrito Federal. La muestra se seleccionó por conveniencia; se incluyeron 100 pacientes diabéticos mayores de 65 años hospitalizados en el Servicio de Medicina Interna en una unidad de segundo nivel de atención, a quienes se aplicaron, a través de observación e interrogatorio directo, la escala Mini-Mental de Folstein (MMSE), la cual evalúa la función cognitiva, y el cuestio- 
nario de polifarmacia, que determina el estado de salud y polifarmacia, ambos validados por consenso de expertos. Existe un alto porcentaje de deterioro cognitivo en pacientes ancianos diabéticos hospitalizados en medicina interna de una unidad de segundo nivel de atención.

Los resultados fueron analizados a través del programa estadístico SPSS 19.0, con estadística descriptiva. Ya organizados los datos, se mostraron gráficamente y se calcularon cantidades representativas del conjunto o medidas de resumen que los describen, así como medidas de resumen y de dispersión para la descripción de la población.

\section{RESULTADOS}

El 39\% tiene entre 66 y 70 años; predominaron las mujeres en un 65\%; el 55\% de la población tiene un nivel de escolaridad de primaria completa o incompleta; se encuentra casada(o) el 60\%; el $46 \%$ vive con sus hijos; el $84 \%$ se dedica al hogar; $94 \%$ tiene un ingreso económico mensual de 30 o menos salarios mínimos diarios ( $\$ 1,784.00$ o menos); el 90\% tiene más de seis años de evolución con diabetes; $78 \%$ se controla con medicamentos; $51 \%$ presentó polifarmacia; el $76 \%$ de los pacientes considera su estado de salud regular y el $21 \%$ malo.

El $81 \%$ obtuvo un nivel de deterioro cognitivo, mientras que el 19\% tiene un nivel de cognición normal (Figura 1). El $43 \%$ obtuvo un nivel leve de deterioro cognitivo, el $22 \%$ tiene nivel moderado, el $19 \%$ tuvo un nivel normal de cognición y el $16 \%$ un nivel grave de deterioro cognitivo (Figura 2).

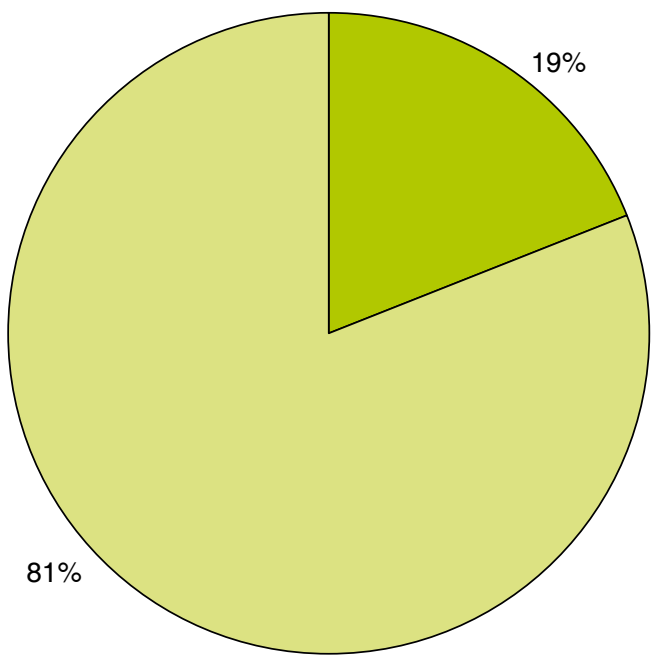

Sin deterioro cognitivo

Con deterioro cognitivo

Figura 1. Deterioro cognitivo.

\section{DISCUSIÓN}

En la muestra estudiada se encontró que el $39 \%$ de los pacientes se ubica en el rango de 66 a 70 años, el $31 \%$ en el de 60 a 65 años, el $20 \%$ corresponde al de 71 a 75 años y el $8 \%$ restante a 80 años y más, encontrando una similitud con el estudio de Mejía A y su grupo, en el que la mayoría de los sujetos se ubicó en el rango de 65 a 74 años y sólo una pequeña porción es mayor de 76 años; ${ }^{3}$ por su parte, Casanova $\mathrm{S}$ concluyó que la prevalencia de deterioro cognitivo es elevada en la ancianidad. ${ }^{4}$

En el estudio realizado por Petersen R y colaboradores se demostró que la prevalencia de deterioro cognitivo decrece con altos niveles de educación. En relación con los años de escolaridad, más de la mitad de la población observada se ubicó en el rango de 0 a 5 años de estudios, lo que coincide con el estudio de Mejía $\mathrm{A},{ }^{3}$ en el que del total de la muestra, el $70 \%$ fue analfabeta o tenía menos de cinco años de escolaridad. Habrá que considerar, además, que en los individuos con niveles más altos de educación se favorece el desarrollo cerebral, el crecimiento dendrítico y la circulación cerebral; también ejecutan mejor las pruebas, retrasando el diagnóstico de deterioro cognitivo. ${ }^{9}$

En el presente estudio, el $69 \%$ de los pacientes no recibe ayuda económica, lo que también concuerda con los resultados de Canazaro M, donde el $86 \%$ de la población tampoco recibe ayuda económica alguna. ${ }^{8}$

Aunado a esto, podemos considerar el costo anual de la persona con diabetes mellitus tipo 2 que presenta complicaciones microvasculares y macrovasculares, y que va de $6,860.00$ a 14,525.00 de pesos mexicanos. La mayoría de la población no cuenta con ingresos para solventar estos costos, lo cual se ha corroborado en la presente investigación, ya que el 94\% de la población estudiada tiene un ingreso mensual de 30 salarios mínimos diarios $(\$ 1,794.00)$ o menos, lo que se traduce en un verdadero problema. ${ }^{10}$

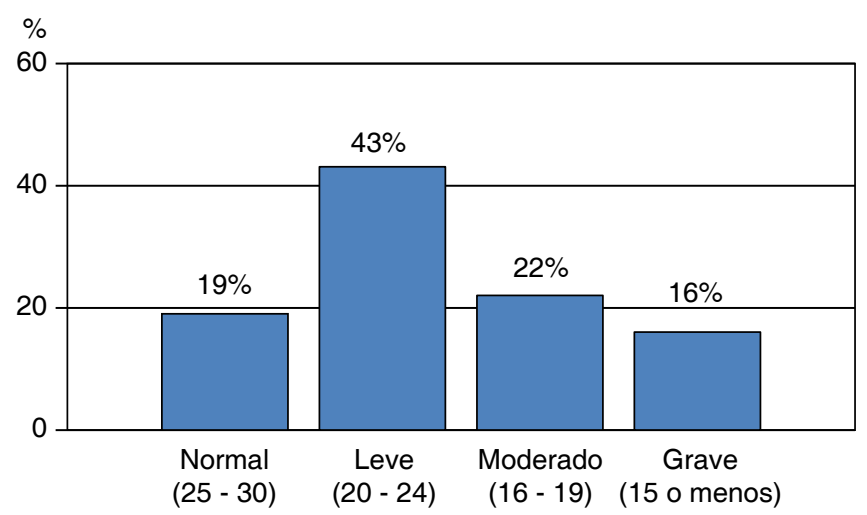

Figura 2. Nivel de deterioro cognitivo. 
Tocante al tiempo de evolución, se encontró que el 25\% de la población tiene un promedio de evolución de la enfermedad de 11 a 15 años, $17 \%$ de 16 a 20 años, el $15 \%$ de 6 a 10 y de 26 a $30,13 \%$ de 21 a $25,9 \%$ tiene 30 o más años de evolución, lo cual es grave. Hassing L, en su estudio "Deterioro cognitivo en pacientes con diabetes tipo 2 e hipertensos", concluyó que los cambios cognitivos se perciben más fácilmente en pacientes ancianos con DMT2 después de seis años de evolución de la enfermedad, en comparación con los no portadores.?

Cabe destacar que los resultados más relevantes radican en que el $81 \%$ de la población tiene deterioro cognitivo y tan sólo el 19\% un nivel de cognición normal; estos datos son consistentes con otros estudios a largo plazo en los que también se utilizó el miniexamen del estado mental (MMSE) para evaluar la función cognitiva en ancianos, que afirman que el aumento en el deterioro cognitivo se relaciona con la diabetes y un alto riesgo de demencia, por lo que se menciona a la diabetes como factor de riesgo en los déficits cognitivos, además de que se ha podido comprobar, en el estudio de Pamplona sobre epidemiología de las demencias, que las personas con hipertensión arterial o diabetes muestran un rendimiento inferior en el examen Mini-Mental de Folstein. ${ }^{10}$

En el presente estudio se concluyó que el $43 \%$ de los pacientes tienen nivel leve de deterioro cognitivo, el $22 \%$ nivel moderado, el $19 \%$ un nivel normal de cognición y el $16 \%$ nivel grave de DC. Comparando los resultados con otros estudios en los que se aplicó la misma escala, se obtuvieron resultados similares, como en el de Ávila ${ }^{11}$ y León, ${ }^{12}$ en el que predominó el DC leve y el resto tuvo un comportamiento similar. En el estudio realizado por Nelson G y su grupo, de acuerdo al grado de severidad del DC, según los resultados de la escala de Hughes se encontró que del total de la población que tuvo DC el $62.9 \%$ obtuvo grado de severidad leve, $18.5 \%$ una severidad moderada y otro $18.5 \%$ una máxima severidad del daño de las funciones corticales superiores. ${ }^{13}$ La DMT2 puede agravar condiciones que se asocian con la dependencia funcional como lo es el deterioro cognitivo, la depresión, los trastornos en la comunicación, en la marcha e incontinencia urinaria, entre otras alteraciones. ${ }^{12}$ Estos pacientes son más propensos a presentar defectos en los procesos cognitivos, especialmente aquellos que no toman las precauciones para su salud y están asociados a cambios isquémicos subcorticales y a la atrofia cerebral progresiva, lo que se pudo observar en el estudio, ya que el porcentaje de pacientes con DC es muy alto. ${ }^{8}$

El panorama es sombrío en cuanto al DC en los ancianos, ya que conduce a que el individuo en edades avanzadas se torne cada vez más vulnerable y tienda a contar con menos probabilidades de recuperación. Estas alteraciones implican una disminución en la capacidad de abstracción, síntesis y generalización del pensamiento; la disminución de la velocidad en las tareas intelectuales, así como en la exactitud y la eficacia, afectan la calidad de vida del individuo.

\section{CONCLUSIONES}

El deterioro cognitivo no es evaluado de forma sistemática en los pacientes diabéticos hospitalizados, por lo que este proyecto dio las pautas para que se llevaran a cabo evaluaciones en el HGR $\mathrm{N}^{\circ} 25$ del Instituto Mexicano del Seguro Social (IMSS) de la Ciudad de México, Distrito Federal, para detectar el grado de deterioro y de esta manera realizar intervenciones enfermeras específicas como: manejo de la hiperglucemia, manejo de la medicación, alimentación, apoyo emocional, enseñanza: actividad/ejercicio prescrito, entrenamiento de la memoria, estimulación cognitiva, facilitar el aprendizaje y mejorar la perfusión cerebral. ${ }^{14}$

Los resultados permiten reflexionar sobre la necesidad de contar con datos fidedignos de los factores que intervienen en el estado cognitivo y funcional del adulto mayor. Esta información nos permite realizar análisis que ayudan a mejorar la atención de enfermería de acuerdo con las condiciones prevalentes en los sistemas de salud mexicanos, a fin de que quienes toman las decisiones elijan las mejores estrategias y diseñen mejores programas para beneficio del adulto mayor.

1. En la muestra de sujetos mayores de 65 años, los resultados de las pruebas verifican la hipótesis de investigación y se rechaza la hipótesis nula, lo que significa que las personas diabéticas presentan un alto porcentaje de deterioro cognitivo.

2. El deterioro cognitivo es una condición importante en la población de adultos mayores con diabetes mellitus tipo 2.

3. La diabetes mellitus tipo 2 puede agravar condiciones que se asocian con la dependencia funcional, como lo es el deterioro cognitivo.

4. Dada la complejidad de la atención del paciente anciano diabético hospitalizado con deterioro cognitivo, el tratamiento hacia éste debe estar basado en una atención multidisciplinaria, para evitar que evolucione a un nivel más severo como la demencia.

5. Las intervenciones de enfermería pueden retardar el proceso de deterioro cognitivo y mejorar la memoria en los ancianos diabéticos. Hoy disponemos de medidas terapéuticas, farmacológicas y no farmacológicas, que pueden mejorar la memoria en ancianos con deterioro cognitivo.

6. Esta investigación sirve de base para generalizar el uso de instrumentos establecidos para explorar la esfera 
cognitiva del anciano diabético y detectar el deterioro cognitivo, fundamentada en evidencia científica y utilización de conceptos y teorías ya establecidos, desarrollando intervenciones de enfermería al anciano diabético con deterioro cognitivo, hospitalizado de acuerdo con la NIC (Nursing Interventions Classification), a través de la valoración, el diagnóstico, la planeación y la ejecución en la que se incluya.

\section{BIBLIOGRAFÍA}

1. INEGI. Censo de Población y Vivienda 2008. Datos de la encuesta Nacional de Salud (Apartado referente a las Enfermedades Crónico Degenerativas a nivel Nacional). México: INEGI; 2008.

2. Gutiérrez M. Teorías del desarrollo cognitivo. España: McGraw Hill; 2005. pp. 10-66.

3. Mejía A, Jaimes A, Villa D, Ruiz A, Gutiérrez R. Deterioro cognoscitivo y factores asociados en adultos mayores en México. Revista de Salud Pública México. 2007; 49 supl 4: 5475-5481.

4. Casanova C. Estudio clínico de las principales causas de trastornos cognoscitivos en la atención primaria. Rev Cubana Med Gen Integr. 2001; 17 (4): 309-315.

5. Koivisto K, Reinikainen K, Haninen T, Vanhanen M, Helkala EL, Mykkanen L et al. Prevalence of age-associated memory impairment in a randomly selected population from eastern Finland. Neurology. 1995; 45 (4): 741-747.

6. Petersen R, Doody R, Kurz A, Mohs R, Morris J, Rabins P et al. Current concepts in mild cognitive impairment. Arch Neurol. 2001; 58: 1985-1992.

7. Hassing L, Scott M, Sven E, Stig B, Pedersen L. Comorbid type 2 diabetes mellitus and hypertension exacerbates cognitive decline: evidence from a longitudinal study. Age and Ageing. 2004; 33: 355.

8. Canazaro de M, Fernández L, Cunhas S, Lima A. El deterioro cognitivo en pacientes ancianos con diabetes mellitus tipo 2 y la identificación de formas de prevención. Rev Neuropsicológica. 2010; 10 (2): 29-42.

9. Ham C, Gutiérrez R. Salud y envejecimiento en el siglo XX. Rev Sal Pub. 2007; 49 (4): 433-435.

10. Noxpanco P. Influencia de la salud-competencia familiar en el autocuidado y nivel de conocimientos del paciente con diabetes mellitus tipo 2 en la Clínica Brimex [Tesis para obtener el título de Licenciado en Enfermería]. México: Universidad Nacional Autónoma de México; 2006. pp. 1-30.

11. Ávila O, Vázquez M, Gutiérrez M. Deterioro cognitivo en el adulto mayor. Ciencia. 2007; 42 (4): 43.

12. León A, Milián S, Camacho C, Arévalo C, Escartín C. Factores de riesgo para deterioro cognitivo y funcional en el adulto mayor. Rev Med Inst Mex Seguro Soc. 2009: 47 (3): 277-284.

13. Gómez V, Bonnin R, Molina, Yáñez F, González Z. Caracterización clínica de pacientes con deterioro cognitivo. Rev Cubana Med. 2003, 42 (1): $12-17$.

14. Heather HT. Diagnósticos Enfermeros: Definiciones y Clasificación 2009-2011. Barcelona: Elsevier, España; 2010. pp. 155-177. 